\title{
Upgrade your practice management
}

Carestream Dental is delighted to announce that it's famed practice management software, CS R4+, has recently upgraded to version 7.0.

This new upgrade not only refreshes the software visually, but also provides a

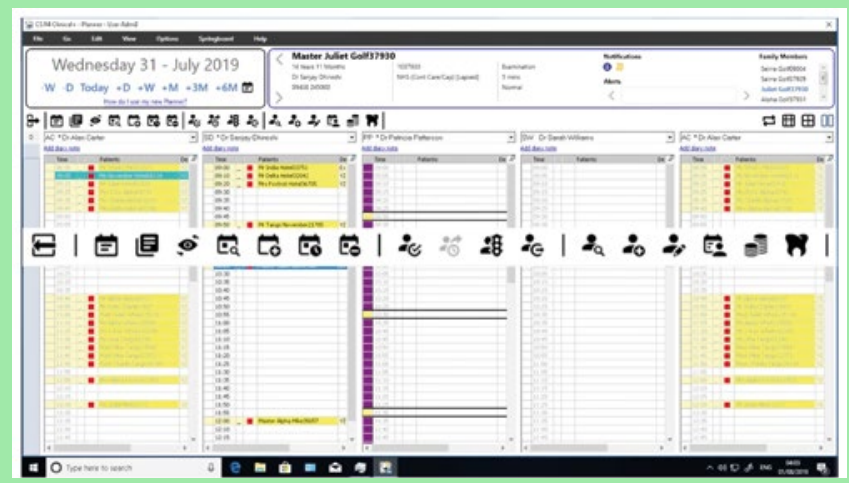

number of innovative new features.

One of these is the Parking Lot function.

When making and changing appointments, users can now drag and drop appointments to new time slots with ease, eliminating the need to re-enter information each time.

This allows the system to keep up to pace with the constantly changing aspects of a modern dental practice.

To find out more, contact Carestream Dental on 0800169 9692 or visit www. carestreamdental. co.uk.

\section{Recipe for success}

Results are everything in implantology. To optimise your chances of treatment success, you need a system that encompasses all the relevant components - you need the Z1.

The result of 15 years of research, the $\mathrm{Z} 1$ offers biocompatibility, mechanical resistance, primary stability and longevity, with an average success rate of $98.6 \%$.

Plus, because it's universal and suits all types of prosthetic restorations (including traditional or CAD/CAM manufactured solutions), you don't need to worry about changing between systems enabling you to dedicate your time to more important matters at hand.

For more information on the Z1 implant, visit tbr.dental, email support@denkauk.com or call 08007076212 .

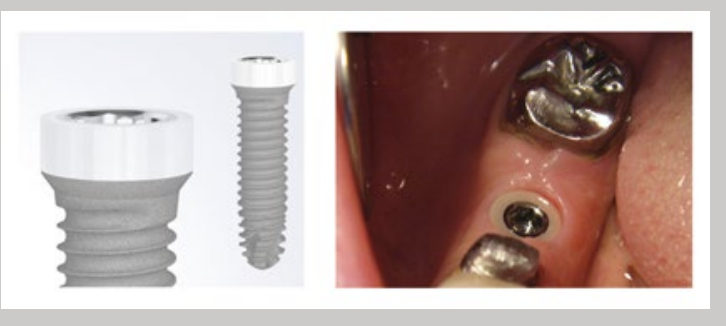

\section{Commitment is to quality}

TANDEX believes that the perfect product has to start with the highest quality raw materials. That's why its range of brushes and adjuncts are free from phthalates, PVC, bisphenol-A and other hormone disrupting or harmful substances.

From its headquarters in Copenhagen, TANDEX has complete control over manufacture and production. This means it has been able to develop a range of preventive tools which are consistent with what patients need to maintain optimal oral health.
From the FLEXI interdental brush to PREVENT GEL, all products are enjoyable to use, too.

For more information on Tandex's range of products. visit www.tandex.dk.

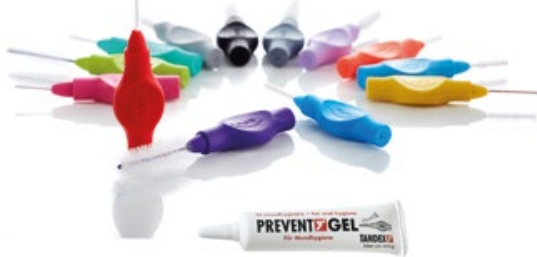

Take the hard work out

Clark Dental offers the first purpose-built, web-based software for digitally recording dental radiographic quality assurance audits as part of regulatory protocols. X-Ray QA provides various guides and tools that are designed to help you achieve and maintain the highest quality radiographs.

Suitable for all types of imaging systems, X-Ray QA can be used to create customised audits and professional reports. It also enables you to track results and identify potential issues instantly, whilst providing a platform for comparing audits and monitoring your progress over time.

Discover how X-Ray QA takes all the hard work out of dental radiographic auditing by contacting Clark Dental on 01268733 146, email info@clarkdental. co.uk or visit www.clarkdental.co.uk.

\section{A new introduction}

Help patients manage acute dental problems by recommending Perio Plus+ mouth rinse. This revolutionary new product offers the benefits of natural ingredients, proving to be more effective than market-leading chlorhexidine (CHX) solutions, thanks to its unique formula.

Perio Plus+ not only contains CHX, but also CITROX - a naturally-derived substance that boasts potent anti-microbial, anti-oxidant, and anti-inflammatory properties. CITROX is combined with polylysine amino acids for enhanced efficacy and prolonged working time.

Moreover, Perio Plus+ ensures compliance through pleasant taste and minimal adverse side effects typically associated with traditional mouth rinses. This makes it a highly valuable supplement to routine tooth brushing and interdental cleaning.

For more information call 01480 862084, email info@curaprox.co.uk or visit www.curaprox.co.uk.

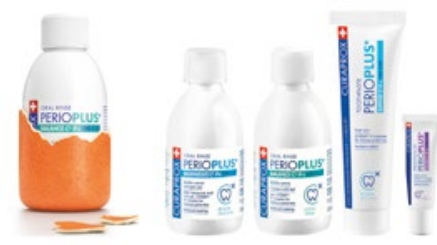

